\title{
Análisis de la influencia de la calidad del contacto de los sensores en el análisis de vibraciones
}

\section{Analysis of the influence of the quality of the contact of the sensors in the vibration analysis}

Wilson Alejandro Huilca Álvarez. ${ }^{1}$

Recibido: 17-05-2019 / Revisado: 19-06-2019 /Aceptado: 07-07-2019/ Publicado: 25-07-2019

\begin{abstract}
.
DOI: https://doi.org/10.33262/cienciadigital.v3i3.2.1.779

The maintenance engineering career has achieved a great evolution in the last decade especially in the science of maintenance based on the condition that allows to control the operating parameters of an asset.

In the different types of industries that exist worldwide there are machines that work by an electric motor, this being a universal equipment, to ensure the desired operation of electric motors one of the main maintenance tasks based on the condition is the measurement of vibrations in the motor for which it must be taken into account that when making vibration measurements a rigid part should be found where the sensors can be placed, in view of the lack of rigid points, the use of small plates has been considered. metal called inserts which will be placed on top of the motor in the place of the bolts, using studs that will adjust the insert to the motor housing and allow a flat surface where the sensor can be placed without any problem. The use of inserts when making vibration measurements directly affects the quality of the wave obtained, allowing measurements to be more accurate.
\end{abstract}

Keywords: Maintenance based on the condition, operating parameters of an asset, inserts, studs

\section{Resumen.}

La carrera de ingeniería de mantenimiento ha logrado una gran evolución en la última década especialmente en la ciencia del mantenimiento basado en lacondición que permite llevar el control de los parámetros de funcionamiento de un activo.

1 Escuela Superior Politécnica de Chimborazo, Facultad de Mecánica. Riobamba, Ecuador. wilson.huilca@espoch.edu.ec 
En las diferentes tipos de industrias que existen a nivel mundial existen maquinas que funcionan mediante un motor eléctrico, siendo este un equipo universal, para asegurar el funcionamiento deseado de los motores eléctricos una de las principales tareas de mantenimiento basado en la condición es la medición de vibraciones en el motor para lo cual se debe tomar en cuenta que al momento de realizar mediciones de vibraciones se debe buscar una parte rígido donde se pueda colocar los sensores, en vista de la falta de puntos rígidos se ha planteado el uso de pequeñas placas de metal llamados insertos los cuales se colocara en la parte superior del motor en el lugar de los pernos, usando pernos prisioneros que ajustaran el inserto a la carcasa del motor y permitirán una superficie plana donde se pueda colocar el sensor sin problema alguno. El uso de insertos al momento de realizar las mediciones de vibraciones afecta directamente a la calidad de la onda obtenida, permitiendo que las medidas sean de mayor precisión.

Palabras claves: mantenimiento basado en la condición, parámetros de funcionamiento de un activo, insertos, pernos prisioneros.

\section{Introducción.}

El principal objetivo del mantenimiento es garantizar las metas de la empresa principalmente la confiabilidad de un activo físico (Parra \& Crespo 2012). Para garantizar las metas del mantenimiento se han desarrollado diferentes metodologías que se ajustan de acuerdo a las políticas de cada tipo de empresa, siendo el monitoreo basado en la condición una de las metodologías más efectivas al momento de asegurar las funciones y los estándares de funcionamiento de un activo (Rodríguez 2014).)

Los diferentes tipos de empresas tienen algún tipo de maquina rotativa impulsada por un motor eléctrico que varía en las dimensiones según la necesidad, pero conservando los mismos principios de funcionamiento. Entre las tareas basadas en la condición de mayor importancia en las industrias es la medición de las vibraciones en los motores eléctricos que permite diagnosticar los modos de fallos (Castellino, Donolo, Bossio, De Angelo, \& García 2007).

Una vibración es un movimiento oscilatorio o movimiento repetitivo de un objeto, la vibración es una combinación de movimiento los cuales hacen que el objeto se mueva en la misma dirección en cualquier momento. Las vibraciones permisibles en una maquinan dependen de los parámetros de operación que son definidos por el usuario (Acosta \& Carrillo 2007).

La norma ISO 20816-1: 2016 establece los parámetros necesarios para medición y evaluación de la vibración de la máquina, en donde se encuentran las directrices generales para la evaluación de vibraciones realizando mediciones en las partes rotativas, no rotativas y no recíprocas de máquinas completas, los valores obtenidos serán utilizados para determinar en qué condiciones se encuentra la maquina además establece rangos de 
velocidad y frecuencia limite dependiendo de la clase de maquina a la cual se realice la medición de vibración. (ISO 20816-1 2016)

En el momento del diseño y construcción de los motores eléctricos los fabricantes no conocen las metodologías de mantenimiento que realiza cada empresa, por tanto, los motores no tienen superficies donde se coloquen los sensores y para ello los inspectores de vibraciones utilizan piezas metálicas o llamados también insertos que se fabrican de acuerdo a la necesidad de cada sensor. (Ortega 2007).

La calidad de las mediciones de las vibraciones están relacionadas directamente con los puntos donde se van a realizar las mediciones y a la rigidez por lo que se plantea mejorar la calidad de la onda medida colocando insertos en los motores eléctricos que permitan tener una área mayor y con una rigidez mayor donde se pueda colocar el sensor para que las medidas que se obtienen sean de mayor exactitud lo que permite identificar de mejor manera que fenómenos se encuentran presentes en el activo y definir las acciones que se consideran necesarias para minimizar las consecuencias de los fallos funcionales (Lanata 2009).

Las mediciones de vibración se las realiza con la finalidad de obtener datos que son necesarios para realizar las conclusiones del monitoreo (Ocampo 2013). Un control en los parámetros de funcionamiento o condiciones de funcionamiento de un activo tiene relación con la reducción de los costos por mantenimiento correctivos e incrementa la disponibilidad del activo (García \& Rodriguez 1998)

Existen diferentes tipos de sensores para medir vibraciones entre los cuales se pueden mencionar:

Transductores de medición de vibración: Son sensores elaborados y empleados para medir velocidad lineal, desplazamiento, proximidad y además son capaces de medir la aceleración, sometidos en la vibración de un motor (Pernia 2004). Estos sensores vienen presentados de diferente forma pueden ser simples, encapsulados, o ser parte de un instrumento de medida. Para una correcta selección se hace de acuerdo a una serie de características las cuales son las siguientes: Rango de medición, rango de frecuencia, precisión sensibilidad transversal y condiciones ambientales (Espinosa 2012)

Acelerómetros: Son los dispositivos más utilizados para el análisis de vibraciones colocados sobre una base. Son sensores que nos permiten medir la vibración y aceleración, los mismos que transforman la aceleración de gravedad en una señal eléctrica analógica, estos sensores se los ubica únicamente donde exista los rodamientos de las maquinas el cual va a medir la vibración de la carcasa del motor. (Ruedas. R., Hernández U., Aranda H., Nava T. \& Tena A 2017).

Acelerómetros piezoeléctricos: Este tipo de acelerómetros se basan en un principio de funcionamiento de efecto piezoeléctrico. Este efecto toma las propiedades de los cristales 
piezo eléctricos (López \& Pablos, 2003) El significado de la palabra piezo que nombra significa "apretar". Cuando existe la presencia de una fuerza en estos cristales se forma una corriente eléctrica, debido a la variación de su estructura cristalina. Existe una desventaja de estos dispositivos en la cual es la frecuencia máxima de trabajo (Arenas 2008).

El Sensor de Proximidad: El sensor de proximidad es muy común aplicado en la industria. Comercialmente se lo conoce como "Proximiter" también es llamado como "sensor de corriente o remolino" este señor necesita de una fuente de alimentación para que el cual genere una señal. El principio de funcionamiento se basa en un principio magnético por el cual es sensible a anomalías magnéticas en la flecha. Este sensor no tiene incorporado elementos mecánicos en el interior. Este dispositivo se lo coloca en máquinas que contengan cojinetes, se caracteriza por que es pequeño por lo que hay que roscar y atornillar. El proximitor mide el movimiento del eje respecto a la máquina (Díaz 2011).

Transductor de Desplazamiento (Corriente Eddy): Los dispositivos de corriente Eddy se caracterizan por que son dispositivos sin contacto, miden la posición de un eje giratorio con respecto a un punto "fijo". Cuando se realiza el cambio de una posición del eje es donde nos proporciona una indicación de vibración. En la parte de la punta de este dispositivo está conformada por una bobina en donde al recibir una señal de frecuencia elevada es ahí donde se genera un campo electromagnético. Con respecto a la rotación del eje bajo las condiciones de vibración la punta actuara alejándose o acercándose con el campo magnético. La ventaja de utilizar este de tipo de sensor es que puede ser utilizados en campos accidentados ya que nos ofrece simplicidad y confiabilidad de operación (Ramírez, Jiménez \& Carreño 2014).

Sensor de Velocidad: Este tipo de sensor está constituido mediante una bobina móvil fuera de un imán permanente. Otro sensor de velocidad es un acelerómetro incorporado por un integrador electrónico a este se lo conoce como Velocímetro es uno de los más clásicos para medir velocidad. Los cuales fueron los primeros en construir. Este sensor viene incorporado de una bobina de alambre y un imán, por el cual, si se mueve el Carter, el imán tiende a permanecer inmóvil producto de la inercia. El sensor produce una señal directamente proporcional a la velocidad. (Arribas \& Rodríguez 2010).

Transformador Diferencial Variable Lineal (LVDT linear variable differential transformer): Este dispositivo es capaz de producir una salida eléctrica proporcional a su desplazamiento de un núcleo móvil separado. Este sensor tiene incorporado de dos bobinas que se encuentran dentro de un cilindro hueco, este tipo de bobinas se los conoce con el nombre de bobina primaria y bobina secundaria las cuales están a una distancia simétricas (Rodríguez \& Cruz 2012)

Las gráficos de control es una herramienta que se emplea para estar en constante cuidado de los procesos generalmente que conlleva a producción junto con una gran ayuda de 
mejoramiento continuo y verificación de puntos de estimación máximo y mínimos los cuales determina el proceso el cual es el desfase, para ello se utiliza los gráficos de control de calidad; en el artículo se utiliza para controlar la frecuencia natural del motor y comparar el comportamiento de las ondas emitidas por el acelerómetro (del Valle 2017).

\section{Metodologia.}

En esta sección del proyecto ubicaremos los materiales y equipos que son necesarios para la toma de datos en el laboratorio. Los cuales tenemos los siguientes:

Módulo de resonancia, el cual tiene integrado los siguientes equipos; el motor eléctrico y el tablero de control con sus respectivos componentes en él está conectado un potenciómetro el cual controla la intensidad de entrada por consiguiente afecta a la velocidad del motor.

Analizador de vibraciones (VIBRACHECK), el cual se caracteriza porque es muy rápido y confiable para la toma de datos, además es ligero y su tamaño es agradable para su transporte. Es capaz de medir los espectros la aceleración, velocidad.

Foto- Tacómetro, este instrumento de medida nos proporciona la velocidad de rotación del eje del motor, mediante un láser que es apuntado al eje, fue utilizado para medir la velocidad de rotación del eje del motor.

Sensor acelerómetro, con este sensor medimos las ondas de aceleración de la carcasa del motor, el cual previamente es conectado al vibracheck y esté conectado por medio de un cable USB a un computador el cual tiene instalado el programa software Maintrag VIEWER.

Software para análisis de vibraciones, mediante este software obtenemos señales de vibración las cuales serán utilizadas para el análisis vibracional, dichas señales están con sus respectivas medidas de frecuencia y frecuencia fundamental.

Insertos, son accesorios necesarios para la correcta medición de las señales de vibración del motor, de este accesorio depende una medida confiable, su diseño de fabricación serán según las necesidades de trabajo del operario, el material utilizado para la fabricación de este accesorio es acero o también puede ser de hierro dulce.

Esto se realizó en el laboratorio de Mantenimiento Predictivo, y los datos fueron tomados en el módulo de resonancia y se obtuvieron 20 datos, cabe mencionar que estas mediciones se realizado con la ayuda del sensor acelerómetro el cual fue fijado en los insertos colocados en el motor este tipo sensores poseen imanes de neodimio los cuales tiene una fuerza de 60 libras para su agarre, las ventajas por las cuales se utilizó este sensor fue que no se necesita alimentar el sensor, no necesitamos acondicionar la señal este sensor nos arrojan ondas de aceleración. Se debe tener en cuenta que las superficies en las cuales se colocara el sensor acelerómetro deberán ser planas, lisas, limpias. Es por ello que se utilizó insertos los cuales están colocados en las partes en donde existe rodamientos, los acelerómetros no se los deberá colocar en la tapa del ventilador. Las otras 20 mediciones que se realizo fue sin la presencia de insertos. 
Es importante realizar un procedimiento analítico que establezcan las diferencias que existen entre la obtención de datos mediante ayuda de insertos en el motor en lugares fijos y la no utilización de los insertos en la obtención de datos de las vibraciones, además se tomó en cuenta el correcto contacto entre la superficie de los insertos colocados en el motor y la superficie del acelerómetro.

\section{Resultados.}

La calidad del contacto de los sensores se determinó mediante las mediciones obtenidas con el sensor acelerómetro. En la tabla 1 podemos observar los resultados de las 20 mediciones que se realizaron en el motor que poseía insertos. Se puede observar que los valores obtenidos se encuentran en un rango aceptable el cual no afectara la medición de los espectros del motor.

En la tabla 2 podemos observar que los valores obtenidos son mayores en comparación a los valores que fueron obtenidos en la tabla 1.Los valores de la tabla 2 se van incrementando conforme se van retirando los insertos del motor y se vayan tomando las mediciones en las partes que no posean los insertos esto afectara la calidad del contacto entre el sensor acelerómetro y las partes del motor que van a hacer medidas, esto nos dice que cuando no exista insertos colocados en el motor los datos obtenidos no serán $100 \%$ confiables debido a la distorsión que ocasiona realizar mediciones sin insertos.

Tabla 1. Mediciones realizadas con insertos

\begin{tabular}{lclc}
\hline \multicolumn{4}{c}{ Mediciones con insertos } \\
\hline \multicolumn{4}{c}{} \\
Nro & Fracción de defectos & Nro & Fracción de defectos \\
1 & 66.762 & 11 & 60.721 \\
2 & 62.216 & 12 & 63.396 \\
3 & 67.945 & 13 & 62.157 \\
4 & 61.874 & 14 & 67.435 \\
5 & 63.453 & 15 & 64.299 \\
6 & 63.382 & 16 & 66.029 \\
7 & 60.347 & 17 & 65.26 \\
8 & 60.241 & 18 & 62.138 \\
9 & 67.4 & 19 & 62.508 \\
10 & 61.163 & 20 & 65.46 \\
\hline
\end{tabular}

Elaborado por: Alejandro Huilca 
Tabla 2. Parámetros de la gráfica de control por la fracción de defectos

\begin{tabular}{|c|c|c|c|c|c|}
\hline \multirow[b]{3}{*}{ Nro } & FRACCION & \multicolumn{4}{|l|}{ FRACCION } \\
\hline & DE & DE & DESVIACIÓN & & \\
\hline & DEFECTOS & DEFECTOS & ESTANDAR & $\mathrm{LCl}$ & LCS \\
\hline 1 & 66.762 & 74,874 & 2,480442917 & 56,2679712 & 71,1506288 \\
\hline 2 & 62.216 & 90,875 & 2,480442917 & 56,2679712 & 71,1506288 \\
\hline 3 & 67.945 & 84,785 & 2,480442917 & 56,2679712 & 71,1506288 \\
\hline 4 & 61.874 & 84,06 & 2,480442917 & 56,2679712 & 71,1506288 \\
\hline 5 & 63.453 & 87,301 & 2,480442917 & 56,2679712 & 71,1506288 \\
\hline 6 & 63.382 & 84,865 & 2,480442917 & 56,2679712 & 71,1506288 \\
\hline 7 & 60.347 & 85,667 & 2,480442917 & 56,2679712 & 71,1506288 \\
\hline 8 & 60.241 & 84,632 & 2,480442917 & 56,2679712 & 71,1506288 \\
\hline 9 & 67.4 & 87,231 & 2,480442917 & 56,2679712 & 71,1506288 \\
\hline 10 & 61.163 & 83,42 & 2,480442917 & 56,2679712 & 71,1506288 \\
\hline 11 & 60.721 & 83,409 & 2,480442917 & 56,2679712 & 71,1506288 \\
\hline 12 & 63.396 & 85,393 & 2,480442917 & 56,2679712 & 71,1506288 \\
\hline 13 & 62.157 & 84,711 & 2,480442917 & 56,2679712 & 71,1506288 \\
\hline 14 & 67.435 & 87,698 & 2,480442917 & 56,2679712 & 71,1506288 \\
\hline 15 & 64.299 & 87,651 & 2,480442917 & 56,2679712 & 71,1506288 \\
\hline 16 & 66.029 & 83,669 & 2,480442917 & 56,2679712 & 71,1506288 \\
\hline 17 & 65.26 & 84,832 & 2,480442917 & 56,2679712 & 71,1506288 \\
\hline 18 & 62.138 & 85,362 & 2,480442917 & 56,2679712 & 71,1506288 \\
\hline 19 & 62.508 & 87,919 & 2,480442917 & 56,2679712 & 71,1506288 \\
\hline 20 & 65.46 & 87,68 & 2,480442917 & 56,2679712 & 71,1506288 \\
\hline
\end{tabular}

Elaborado por: Alejandro Huilca 


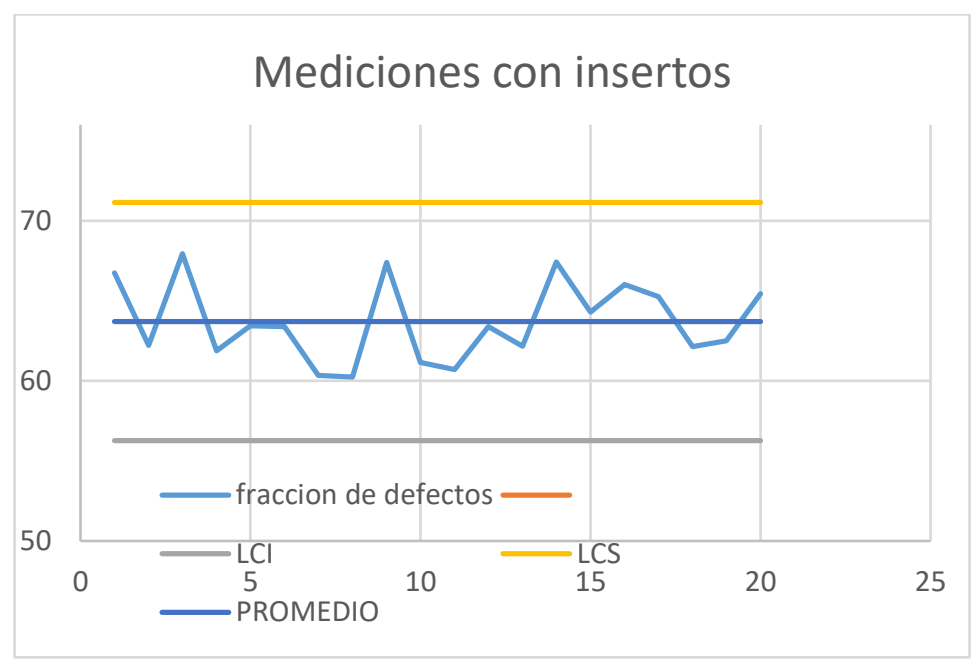

\section{Gráfica 1. Gráfica de control, mediciones con insertos}

Elaborado por: Alejandro Huilca

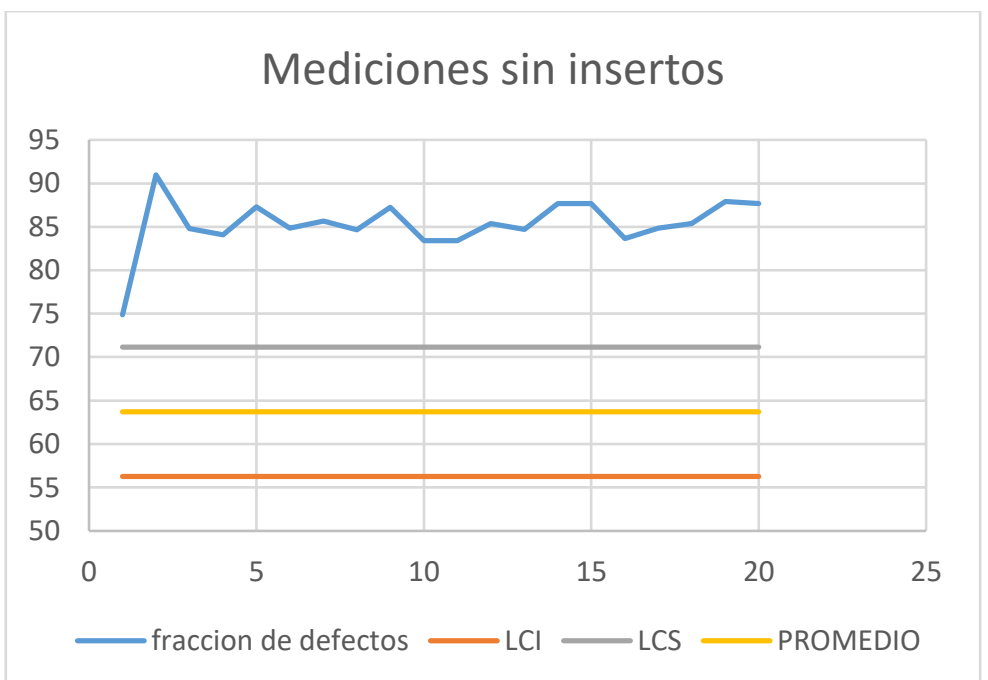

Gráfica 2. Gráfica de control, mediciones son insertos

\section{Elaborado por: Alejandro Huilca}

\section{Discusión}

Los resultados obtenidos muestran evidencia a favor del uso de los insertos, debido a que los valores obtenidos parecen tener una mayor calidad en los contactos del sensor con los insertos en comparación a los demás datos obtenidos sin la presencia de insertos los cuales no se encontraban en el límite superior de la gráfica de control antes analizada. Cabe mencionar que si bien los valores tomados con insertos se encuentran en los rangos de la gráfica tanto inferior como superior la eficacia de todos estos datos dependerá mucho de la manipulación del acelerómetro y la posición en la cual se realizará la medición.

En las mediciones obtenidas sin insertos llama mucho la atención, que los valores obtenidos no se encuentren en los rangos limites superior e inferior de la gráfica de control, ya que muchos 
técnicos que realizan sus mediciones no utilizan los insertos y los resultados que presentan son erróneos , pero ellos desconocen el motivo por el cual los datos obtenidos no son los reales, es así que podemos concluir en ese sentido que para que los datos sean correctos y nos ayuden a diagnosticar las fallas que poseen los equipos (motores eléctricos) se deberá utilizar los insertos para realizar las mediciones. A nuestro criterio lo que ocurre cuando no se utiliza los insertos es que los datos que son obtenidos son perturbados con el movimiento de la máquina y no permite un contacto adecuado del sensor acelerómetro por los que se recomienda el uso de los insertos para realizar las mediciones.

en una metodología llamada graficas de control es ahí donde se puede ver la calidad de la toma de datos, en dicha grafica establecemos, tanto un valor superior y valor inferior si están dentro de ese rango es una toma de datos correcto y por lo contrario son tomas de datos incorrectos, es así que finalmente se pudo apreciar gráficamente que la toma de datos que están dentro del rango establecido es con la presencia de insertos.

Por lo tanto, el análisis de vibración de la máquina será idónea en donde se puede identificar los fallos presentes en dicha máquina.

\section{Conclusiones}

- En conclusión, esta investigación se basó en la importancia de colocar insertos en las maquinas, y sin insertos de la misma máquina a analizar en la cual se representó en una metodología llamada graficas de control es ahí donde se puede ver la calidad de la toma de datos, en dicha grafica establecemos, tanto un valor superior y valor inferior si están dentro de ese rango es una toma de datos correcto y por lo contrario son tomas de datos incorrectos, es así que finalmente se pudo apreciar gráficamente que la toma de datos que están dentro del rango establecido es con la presencia de insertos.

- Por lo tanto, el análisis de vibración de la máquina será idónea en donde se puede identificar los fallos presentes en dicha máquina.

\section{Referencias Bibliográficas}

Acosta Tamayo, J. F., \& Carrillo Tamayo, D. H. (2007). Diseño y construcción de una máquina clasificadora por diámetro para champiñones con capacidad de 1 ton/día para la empresa KENNET. Tesis de grado ESPE, Sangolquí

Arenas, M. (2008). Sistema Para la Adquisicion y monitorizacion de aceleracion mediante microprocesador. Universidad de Sevilla, 39-54.

Arribas, M. T., \& Rodríguez, M. M. (2010). Análisis de vibraciones en auxiliares de MAC1. Zaragoeza

Castellino, A. M., Donolo, P. D., Bossio, G. R., De Angelo, C. H., \& García, G. (2007). Diagnóstico de fallas en los rodamientos de motores eléctricos empleando variables eléctricas. XII Reunión de Trabajo en Procesamiento de la Información y Control, 6.

Espinosa, D. A. R. (2012). Trabajo de Diploma. La Planeación Financiera a corto plazo 
en la Empresa Labiofam SA de Las Tunas.

Del Valle Veliz, J. A. (2017). Sistema de control de calidad en la fabricación de toallas de algodón basado en el control estadístico de procesos (Doctoral dissertation, Universidad de San Carlos de Guatemala).

Díaz, S. (2011). Medición y análisis de vibraciones. Laboratorio de dinamica de maquinas

Lanata Rospigliosi, M. Á. W. (2009). Modelamiento de vibraciones en el campo cercano aplicado a lagunas norte.

López, A. C., \& Pablos, R. R. (2003). Materiales inteligentes I/II. In anales de mecánica y electricidad.

Parra, C., \& Crespo, A. (2012). Ingeniería de Mantenimiento y Fiabilidad aplicada a la Gestión de Activos. INGECON

Pernia-Márquez, D. (2004). Introducción a la medición de vibración. Facultad de Ingenierıa. Universidad de los Andes. Venezuela.

Ocampo, J. R. (2013). Sistema de bajo costo para monitoreo de disturbios de vibración en máquinas rotatorias. Innovare: Revista deficiencia y tecnología, 2(2), 14-31.

Ramírez, L. G. C., Jiménez, G. S. A., \& Carreño, J. M. (2014). Sensores y actuadores. Grupo Editorial Patria.

Rodriguez P., \& CRUZ L. (2012). Diseño de un sistema para medir la circularidad de una pieza en la operación de cilindrado (Doctoral dissertation, Universidad Tecnológica de Pereira. Facultad de Tecnologías. Tecnología en Mecatrónica).

Ruedas. R., Hernández U., Aranda H., Nava T. \& Tena A (2017). Instrumentación de equipos de las subestaciones eléctricas: análisis de las vibraciones por operación y vibración forzada. 
PARA CITAR EL ARTÍCULO INDEXADO.

Huilca Álvarez, W. A. (2019). Análisis de la influencia de la calidad del contacto de los sensores en el análisis de vibraciones. Ciencia Digital, 3(3.2.1), 20-30. https://doi.org/10.33262/cienciadigital.v3i3.2.1.779

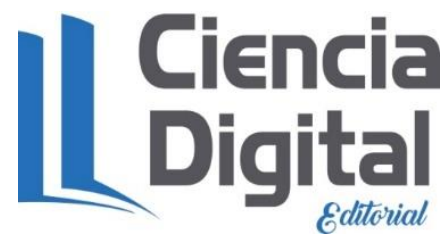

El artículo que se publica es de exclusiva responsabilidad de los autores y no necesariamente reflejan el pensamiento de la Revista Ciencia Digital.

El artículo queda en propiedad de la revista y, por tanto, su publicación parcial y/o total en otro medio tiene que ser autorizado por el director de la Revista Ciencia Digital.
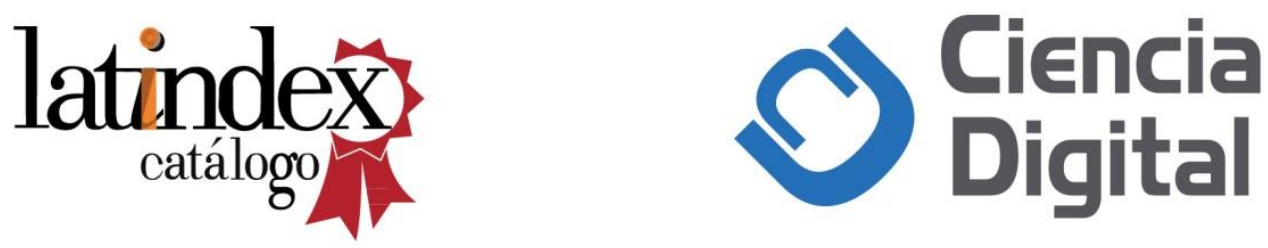